\title{
Experimental study of stable imbibition displacements in a model open fracture. II. Scale-dependent avalanche dynamics
}

\author{
Xavier Clotet, ${ }^{1,2}$ Stéphane Santucci, ${ }^{2, *}$ and Jordi Ortín ${ }^{1, \dagger}$ \\ ${ }^{1}$ Departament ECM, Fac. de Física, Universitat de Barcelona, C. Martí i Franqués 1, 08028 Barcelona, Catalonia, Spain \\ ${ }^{2}$ Laboratoire de physique, CNRS UMR 5672, École Normale Supérieure de Lyon, 46 Allée d'Italie, 69364 Lyon Cedex 07, France
}

(Received 5 September 2015; revised manuscript received 30 November 2015; published 28 January 2016)

\begin{abstract}
We report the results of an experimental investigation of the spatiotemporal dynamics of stable imbibition fronts in a disordered medium, in the regime of capillary disorder, for a wide range of experimental conditions. We have used silicone oils of various viscosities $\mu$ and nearly identical oil-air surface tension, and forced them to slowly invade a model open fracture at very different flow rates v. In this second part of the study we have carried out a scale-dependent statistical analysis of the front dynamics. We have specifically analyzed the influence of $\mu$ and $\mathrm{v}$ on the statistical properties of the velocity $V_{\ell}$, the spatial average of the local front velocities over a window of lateral size $\ell$. We have varied $\ell$ from the local scale defined by our spatial resolution up to the lateral system size $L$. Even though the imposed flow rate is constant, the signals $V_{\ell}(t)$ present very strong fluctuations which evolve systematically with the parameters $\mu, \mathrm{v}$, and $\ell$. We have verified that the non-Gaussian fluctuations of the global velocity $V_{\ell}(t)$ are very well described by a generalized Gumbel statistics. The asymmetric shape and the exponential tail of those distributions are controlled by the number of effective degrees of freedom of the imbibition fronts, given by $N_{\text {eff }}=\ell / \ell_{c}$ (the ratio of the lateral size of the measuring window $\ell$ to the correlation length $\ell_{c} \sim 1 / \sqrt{\mu \mathrm{v}}$ ). The large correlated excursions of $V_{\ell}(t)$ correspond to global avalanches, which reflect extra displacements of the imbibition fronts. We show that global avalanches are power-law distributed, both in sizes and durations, with robustly defined exponents-independent of $\mu, \mathrm{v}$, and $\ell$. Nevertheless, the exponential upper cutoffs of the distributions evolve systematically with those parameters. We have found, moreover, that maximum sizes $\xi_{S}$ and maximum durations $\xi_{T}$ of global avalanches are not controlled by the same mechanism. While $\xi_{S}$ are also determined by $\ell / \ell_{c}$, like the amplitude fluctuations of $V_{\ell}(t), \xi_{T}$ and the temporal correlations of $V_{\ell}(t)$ evolve much more strongly with imposed flow rate $\mathrm{v}$ than with fluid viscosity $\mu$.
\end{abstract}

DOI: 10.1103/PhysRevE.93.012150

\section{INTRODUCTION}

Flows in porous and fractured media exhibit complex spatiotemporal dynamics [1-7]. Avalanches and non-Gaussian intermittent velocity fluctuations [8-17] can arise from the medium heterogeneous structure, which may involve a very wide range of spatial scales, from nanometer pore size to kilometer field scales. A common theoretical approach to study those flows consists in a volume-averaging or homogenization procedure in order to obtain effective behavior at large scale from the up-scaling of microscopic phenomena [18].

We propose here to study the intermittent avalanche dynamics of fluid invasion in a disordered medium as function of the scale of observation of the process. We have indeed developed a model experimental setup which allows accessing in full detail the fluid front motion from the microscopic scale of the heterogeneities up to the system size. Thus, in contrast with most other crackling noise systems [19] where such information is not accessible, we could perform a scale-dependent analysis of the dynamics. In our imbibition experiments, a viscous, wetting oil invades a disordered medium and displaces the air originally present at a constant flow rate v. The medium is a horizontal Hele-Shaw cell with a two-valued gap thickness that fluctuates randomly in space, mimicking an open fracture. Details of the setup and experimental methods are given in Part I (accompanying paper

\footnotetext{
*stephane.santucci@ens-lyon.fr

†ortin@ecm.ub.edu
}

[20]). The imbibition front is distorted by local capillary pressure fluctuations experienced by the meniscus and by fluctuations of the permeability of the medium, both produced by the randomly varying gap thickness of the cell. The viscosity contrast and the interfacial tension between invading and displaced fluid, in contrast, tend to restore front flatness. As a result, the viscous fluid interface develops long-range correlations, quantified by the characteristic length scale $\ell_{c}=$ $\sqrt{\kappa / \mathrm{Ca}}$, where $\kappa$ is the permeability of the medium and $\mathrm{Ca}$ the capillary number of the flow. This number characterizes the relative effect of viscous and capillary forces, $\mathrm{Ca}=\mu \mathrm{v} / \sigma$, with $\mu$ the dynamic viscosity of the invading fluid, $\sigma$ the interfacial tension at the oil-air interface, and $\mathrm{v}$ the mean velocity of the front. Since $\ell_{c}$ diverges when $\mathrm{Ca} \rightarrow 0$, the capillary number provides a quantitative measure of the distance to criticality in imbibition displacements. Indeed, as shown in detail in Part I (accompanying paper [20]), the front dynamics is driven by spatially localized avalanches with various scaling relations expected when the critical point for depinning is approached as $\mathrm{Ca} \rightarrow 0[2,5,14,15]$.

In the accompanying paper (Part I [20]) we have studied the front dynamics only at the local scale of our spatial resolution. Here we focus our attention on the front dynamics through increasing length scales. To this purpose we compute a global velocity $V_{\ell}(t)$ as the spatial average of the local front velocities over a window of lateral size $\ell$, and vary $\ell$ from the local scale defined by our spatial resolution up to the lateral system size $L$. Even though the imposed flow rate is constant, the signals $V_{\ell}(t)$ exhibit strong fluctuations, which evolve systematically with the parameters $\mu, \mathrm{v}$, and $\ell$. We 
have verified that the fluctuations of $V_{\ell}(t)$ are non-Gaussian $[11,14]$. This is a generic property of the fluctuations of spatially averaged (or global) quantities in spatially correlated systems. Non-Gaussian fluctuations are a consequence of the nonapplicability of the central limit theorem to correlated systems [21-24]. The large and jerky excursions of $V_{\ell}(t)$ above its mean value correspond to global avalanches, which reflect extra displacements of the imbibition fronts.

In comparison to our earlier investigations of avalanche dynamics in this setup $[14,15]$ we have used for the first time oils of different dynamic viscosities, from $\mu=10$ to $350 \mathrm{cP}$, without a significant change of surface tension $(\sigma \simeq$ $21 \mathrm{mN} / \mathrm{m}$ ). This effort has led us to discover that the dynamics of $V_{\ell}(t)$ is not simply controlled by the capillary number $\mathrm{Ca} \sim \mu \mathrm{v}$. In addition, the range of imposed flow rates has been sensibly increased to produce mean front velocities in the range $0.04<\mathrm{v}<0.6 \mathrm{~mm} / \mathrm{s}$. In this way we have been able to expand the range of capillary numbers to $6 \times 10^{-5}<$ $\mathrm{Ca}<2 \times 10^{-3}$, and thus we have been able to characterize the different scalings with higher confidence. In order to have highly reliable statistics, finally, we have performed 15 to 20 experiments with different disorder realizations for each experimental condition $(\mathrm{v}, \mu)$.

The remainder of the paper is organized as follows. We study the temporal fluctuations and correlations of $V_{\ell}$ in Sec. II and its avalanche dynamics in Sec. III. Finally, in Sec. IV, we draw the main conclusions of our investigation.

\section{SPATIALLY AVERAGED VELOCITIES}

The spatiotemporal correlations of the local velocities of the front studied in the accompanying paper (Part I [20]) lead to a burstlike dynamics that can be made apparent by plotting the instantaneous velocity of every point of the front within the measurement window in the course of time. Figure 1 gives examples of activity maps $v(x, t)$ for experiments with the same flow rate but different oil viscosities. Our high-resolution setup allows us to measure the velocity of the front at scales much smaller than its lateral correlation length $\ell_{c}$.

Detailed local information is not accessible in most systems that exhibit also a burstlike dynamics. In those systems a spatially averaged signal over the sample (a crackling noise [19]) is studied instead. Having access here to $v(x, t)$, however, allows us to explore systematically the dynamics through increasing length scales. The spatially averaged (sometimes called global) velocity of the front on scale $\ell$ is obtained by averaging the spatiotemporal map $v(x, t)$ of local velocities over a window of lateral size $\ell$ :

$$
V_{\ell}(t)=\frac{1}{\ell} \int_{\ell} v(x, t) d x .
$$

The value of the window size $\ell$ can be varied in a wide range, from the spatial resolution of the images up to the width $L$ of the measurement region (ROI), allowing a scale-dependent statistical analysis of the front dynamics. As a consequence of the jerky dynamics of the front at the local scale (with large fluctuations of $v$ and spatial and temporal correlations) this global signal exhibits also a burstlike dynamics.

We have systematically varied the two controlling parameters $-\mathrm{v}$ and $\mu$-and analyzed the interfaces at different

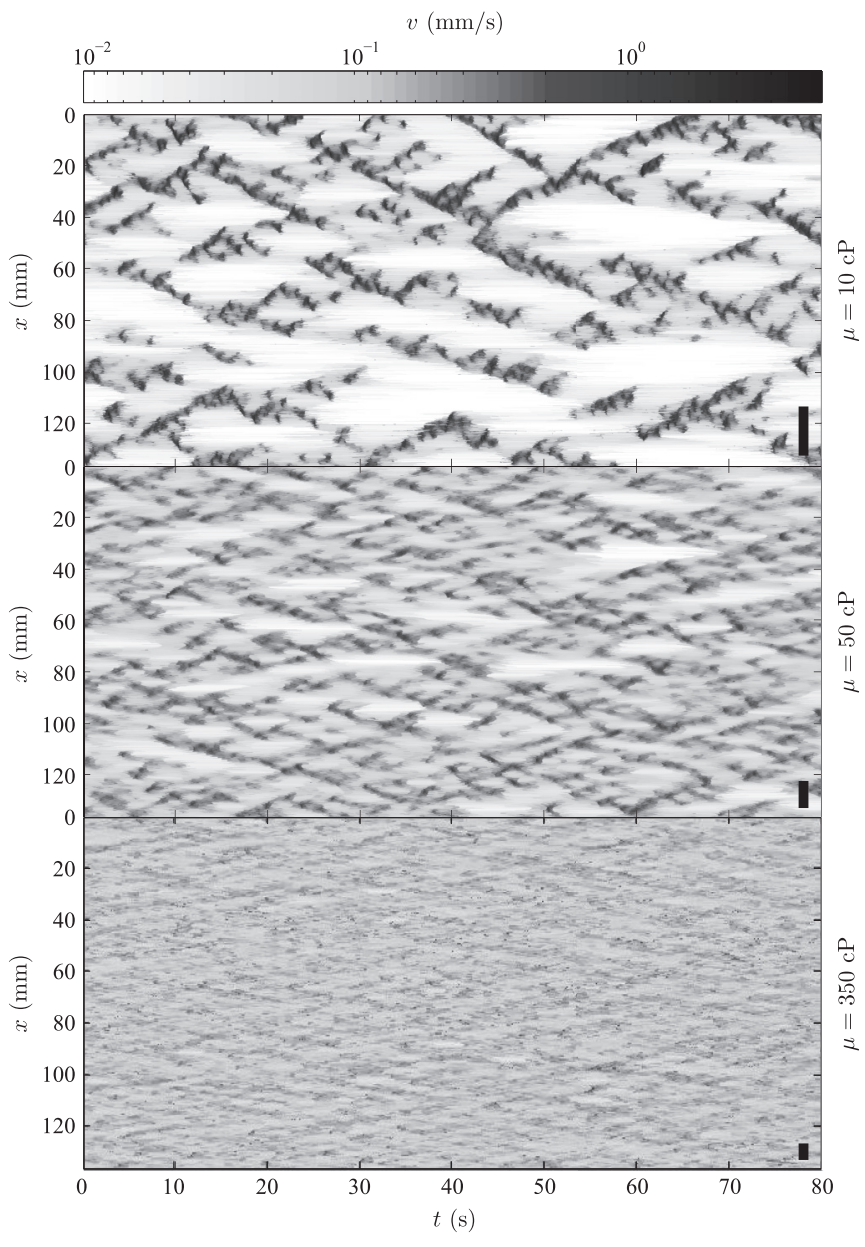

FIG. 1. Spatiotemporal activity maps of the same three experiments shown in Fig. 2 of Part I [20]. $\mathrm{v}=0.13 \mathrm{~mm} / \mathrm{s}$ in all cases but $\mu=10,50,350 \mathrm{cP}$ from top to bottom. The graycode for $v(x, t)$ is the same in all cases. The black vertical line on the bottom right corner of each panel gives the nominal correlation length $\ell_{c}$ for each experimental condition $\left(\ell_{c}=15.3,6.7,2.6 \mathrm{~mm}\right.$ from top to bottom).

windows of observation $\ell$. To qualitatively show the effect of each parameter $(\mathrm{v}, \mu, \ell)$ six typical signals of $V_{\ell}(t)$ are shown in Fig. 2 in groups of two. Each group corresponds to setting two of the parameters constant and changing the third one. In all cases shown, $V_{\ell}(t)$ is a jerky signal that strongly fluctuates around its mean value. The magnitude of the fluctuations is larger when the fluid is injected faster but also when the system is observed at smaller $\ell$ or the fluid has lower viscosity, keeping the other parameters constant. However, the relative magnitude of the fluctuations (compared to the mean velocity of the front) is larger whenever $\ell, \mu$, or $\mathrm{v}$ are smaller and the other parameters are fixed (left panels of the figure). The shape of the fluctuations of the three signals on the left panels (small $\ell, \mu$, or v) looks alike when they are observed at the appropriate scale of velocities. Similarly, the signals on the right also seem to resemble each other. The properties of these signals are analyzed quantitatively in the following.

\section{A. Statistical distributions of $V_{\ell}(t)$}

We first study the amplitude of the fluctuations of the spatially averaged velocity $V_{\ell}(t)$. As recalled in the 


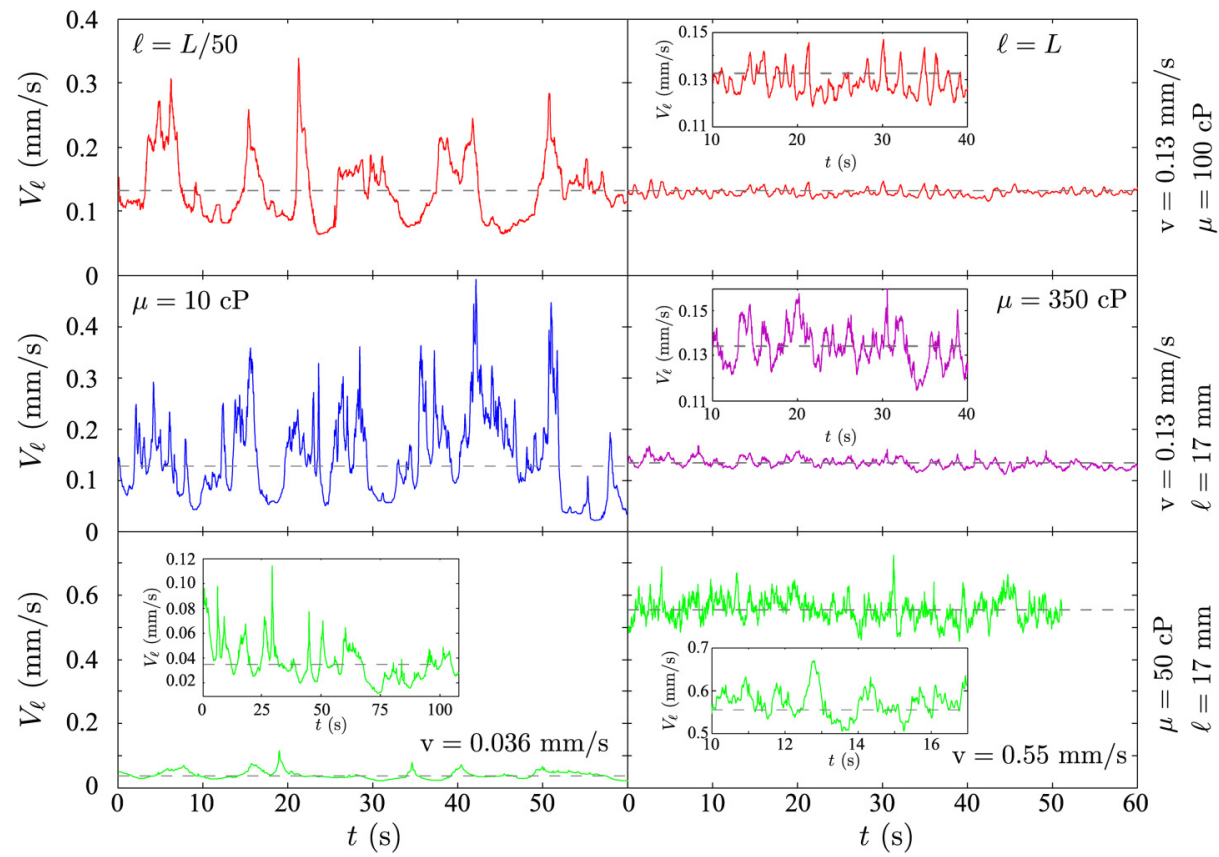

FIG. 2. Examples of spatially averaged (global) velocities of the front as functions of time. Six limiting cases are shown to identify the effect of the window of observation $\ell$ (top), the dynamic viscosity $\mu$ (middle), and the imposed mean velocity $\mathrm{v}$ (bottom) on the actual form of the signal. Each panel (top, middle, bottom) corresponds to setting two of the parameters $(\mathrm{v}, \mu, \ell)$ constant and changing the third one (smaller value on the left than on the right plots). The insets show $V_{\ell}(t)$ corresponding to the same mean advancement of the front $(30 \mathrm{~s}$ at $\mathrm{v}=$ $0.13 \mathrm{~mm} / \mathrm{s})$. Dashed lines indicate the time-averaged velocity $\left\langle V_{\ell}(t)\right\rangle$ in each experiment. System size $L=136 \mathrm{~mm}$.
Introduction and shown in Part I (accompanying paper [20]), local velocities $v(x, t)$ are spatially correlated along $x$ up to a lateral distance given by $\ell_{c}=\sqrt{\kappa / \mathrm{Ca}}$. The global signal $V_{\ell}(t)$ is a spatial average of a spatially correlated signal, and thus the fluctuations of $V_{\ell}(t)$ around its mean are expected to be non-Gaussian [21-24].

Figure 3 shows that this is indeed the case. In this figure we display the probability density function (pdf) of the normalized variable $Y=\left(V_{\ell}-\left\langle V_{\ell}\right\rangle\right) / \sigma_{V_{\ell}}$, where $\left\langle V_{\ell}\right\rangle$ is the temporal average of the signal over the duration of the experiment and $\sigma_{V_{\ell}}$ its standard deviation for different windows of observation $\ell$ (left), dynamic viscosity $\mu$ (middle), or imposed mean velocity $\mathrm{v}$ (right). Those distributions are systematically skewed to values of $V_{\ell}$ larger than the mean in all cases. They depart noticeably from the normal distribution represented by a solid line. Moreover, we can observe that those distribu-

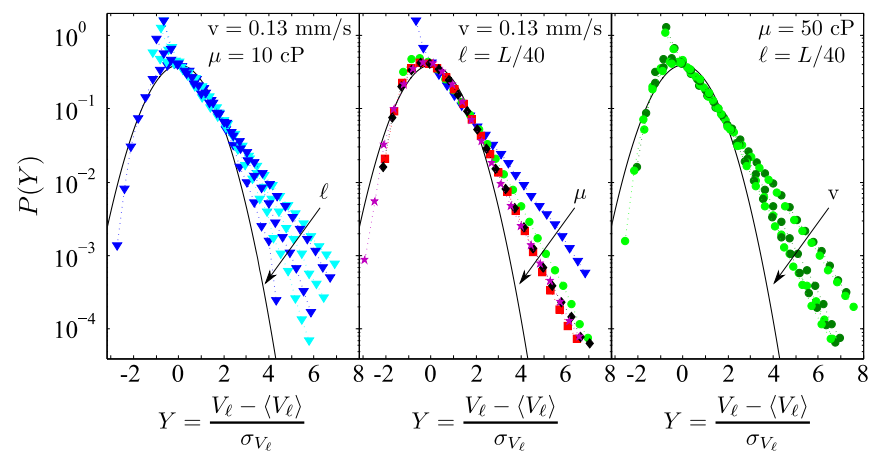

FIG. 3. Evolution of the distributions of the rescaled global velocity with window of observation $\ell$ (left), dynamic viscosity $\mu$ (middle), or imposed mean velocity v (right). In the left panel, data for $\ell=L / n$ with $n=1,8,11,16,27,50$ are plotted. Data in the middle panel correspond to $\mu=10,50,100,169,350 \mathrm{cP}$. Data in the right panel correspond to $\mathrm{v}=0.036,0.053,0.13,0.23,0.35,0.55$ $\mathrm{mm} / \mathrm{s}$. The solid lines correspond to a normal distribution. tions become progressively more and more asymmetric and develop an exponential tail when either the injection rate $\mathrm{v}$ or the viscosity $\mu$ or the window of observation $\ell$ are reduced. The skewness $S k$ of the distributions, plotted as a function of the linear size of the measuring window $\ell$ in Fig. 4, quantifies such asymmetric shape and its evolution with the various experimental conditions studied. In all cases $S k$ is significantly larger than zero at small $\ell$, and it decays monotonously with $\ell$ towards $S k=0$ (symmetric distribution). Also, for a given $\ell$, $S k$ is larger at low v and small $\mu$.

Non-Gaussian fluctuations of spatial averages of correlated variables can arise from the fact that those variables are not statistically independent [21-24]. Hence the central limit theorem does not apply. Various studies have shown that in spatially extended systems, where the correlation lengths are of the order of the system size, the pdf of a global quantity takes under certain conditions a form which is very

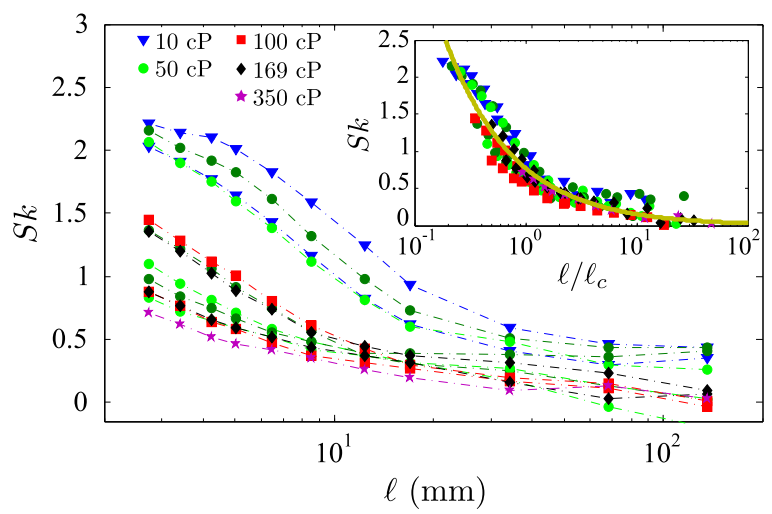

FIG. 4. Skewness of the velocity distributions as a function of $\ell$. Different symbols correspond to different viscosities. The curves obtained for different $\mu$ and $\mathrm{v}$ collapse when plotted as a function of $\ell / \ell_{c}$ (inset). The solid line is a guide to the eye. 
well approximated by generalized Gumbel (GG) distributions [21-26]:

$$
P_{a}(Y)=\frac{a^{a} b_{a}}{\Gamma(a)} \exp \left\{-a\left[b_{a}\left(Y+s_{a}\right)+e^{-b_{a}\left(Y+s_{a}\right)}\right]\right\}
$$

with $b_{a}=\sqrt{\psi^{1}(a)} / \sigma_{Y}$ and $\quad s_{a}=\langle Y\rangle+\left[\ln a-\psi^{0}(a)\right] / b_{a}$, where $\Gamma(a)$ is the gamma function and $\psi^{m}(a)=\frac{d^{m+1}}{d a^{m+1}} \ln \Gamma(a)$ is the polygamma function of order $m$. When the variable $Y$ is normalized, the first two moments of the distribution are $\langle Y\rangle=0$ and $\sigma_{Y}=1$. The only free parameter left to fix the shape of the GG distributions is $a$, related to their skewness by: $S k=\left\langle Y^{3}\right\rangle \sim 1 / \sqrt{a}$. Until now, however, there is no theoretical argument for using the GG distribution in the study of fluctuations of global quantities-except for a particular model that bears no relation with two-phase fluid displacements [22]. In earlier experiments, we showed that the fluctuations of $V_{\ell}(t)$ could be represented by a GG distribution [14]. Those experiments were performed at different $\mathrm{v}$ but using only one oil (of viscosity $\mu=50 \mathrm{cP}$ ). In the present work we have expanded this earlier research in various ways. First, we have increased substantially the range of capillary numbers explored and, thus, of lateral correlation lengths $\ell_{c}$. Second we have included experiments with oils of different dynamic viscosities. This allows us to check the scaling with $\ell / \ell_{c}$ of the GG distributions with respect to both $\mathrm{v}$ and $\mu$ independently.

The asymmetry of the GG distributions is related to the number $N_{\text {eff }}$ of effective degrees of freedom of the system under study [26]. In our case, the effective number of statistically independent domains of the interface can be estimated by the ratio of the window of observation to the lateral correlation length, $N_{\text {eff }}=\ell / \ell_{c}$. Figure 4 shows the skewness $S k=\left\langle Y^{3}\right\rangle$ of the velocity distributions versus $\ell$ for the different oils. For an oil of given viscosity, $S k$ is smaller at larger v. Let us mention here that very large data sets are necessary to compute accurate values of skewness. Considering this difficulty, the inset shows that the skewness of the distributions for the available combinations of experimental parameters reasonably collapse into a common functional form when $S k$ is plotted as function of the reduced variable $\ell / \ell_{c}$. Since $S k$ determines the shape of the distributions of $V_{\ell}(t)$, the effective number of degrees of freedom $N_{\text {eff }}=\ell / \ell_{c}$ appears as the only controlling parameter of the fluctuations of $V_{\ell}(t)$, and $\mathrm{v}$ and $\mu$ play the same role here through $\ell_{c} \sim 1 / \sqrt{\mathrm{v} \mu}$.

To further confirm this result, Fig. 5 displays the statistical distributions of velocity fluctuations for different values of $\ell / \ell_{c}$. Top panels compare the pdfs obtained from separate experiments $(\mu, \mathrm{v})$ analyzed at $\ell / \ell_{c}$ constant. The distributions resulting from considering together all available data with $\ell / \ell_{c}$ constant are also plotted (full symbols). All distributions remarkably collapse. In addition, distributions corresponding to all available data are compared to (not fitted by) GG distributions of same skewness. The agreement is remarkable. Small discrepancies in the high-velocity tails at small scales may be attributed to a lack of statistics for large values of $Y$. Notice, finally, that the distributions are increasingly non-Gaussian as $\ell / \ell_{c}$ decreases.

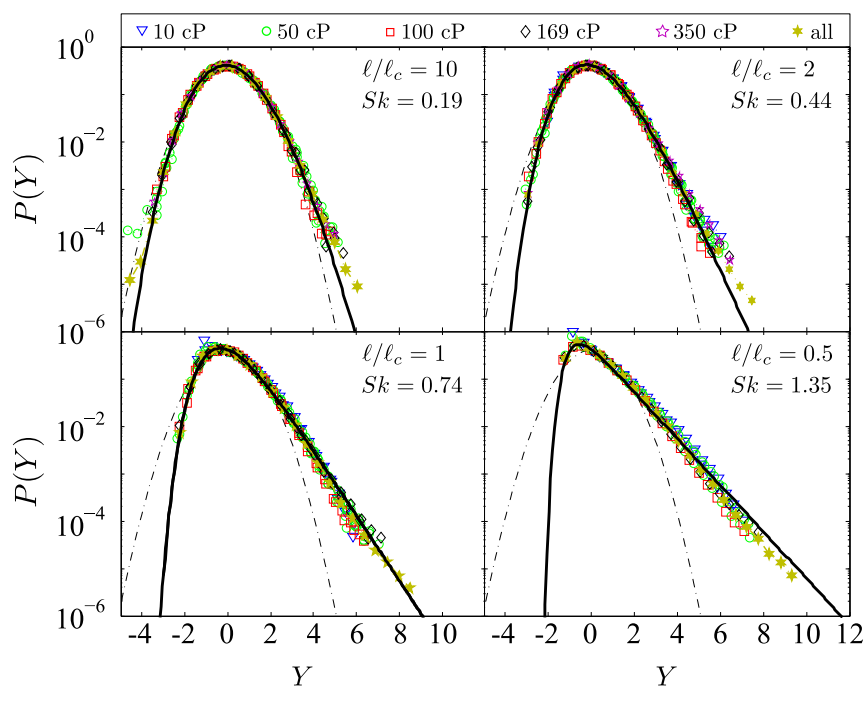

FIG. 5. Distributions of the normalized fluctuations of the global velocity, $Y=\left[\left(V_{\ell}(t)-\left\langle V_{\ell}(t)\right\rangle\right] / \sigma_{V_{\ell}}\right.$, for various values of $\ell / \ell_{c}$. Top panels show $P(Y)$ for all the experiments with different $(\mu, \mathrm{v})$ compatible with $\ell / \ell_{c}=10,2,1$, and 0.5. Distributions for each experiment separately and for data sets containing data from all experiments are shown. Solid lines correspond to generalized Gumbel distributions with the value of $S k$ of the experimental distributions. Dashed-dotted lines correspond to a normal distribution.

\section{B. Temporal correlations of $V_{\ell}(t)$}

We now study the temporal correlations of the fluctuating signal $V_{\ell}(t)$ by computing the autocorrelation function:

$$
C_{V_{\ell}}(\Delta t)=\overline{\left\langle\left(V_{\ell}(t+\Delta t)-\left\langle V_{\ell}(t)\right\rangle\right)\left(V_{\ell}(t)-\left\langle V_{\ell}(t)\right\rangle\right)\right\rangle_{t}} .
$$

Here $\langle\cdots\rangle_{t}$ is an average over the duration of every experiment, and $\cdots$ the ensemble average over different experimental realizations. The results are shown in Fig. 6 for a number of conditions. In all cases $C_{V_{\ell}}$ decays monotonously in time until it reaches a maximum anticorrelation-minimum value of $C_{V_{\ell}}$ below zero-at $\Delta t^{*}$. Above $\Delta t^{*}, C_{V_{\ell}}$ increases towards zero. The temporal correlations of the velocity signal show a systematic evolution with $\ell, \mu$, and $\mathrm{v}$, as displayed on the panels of the figure. The smaller the window of observation, the shorter $\Delta t^{*}$. On the contrary, the maximum anticorrelation is achieved at larger $\Delta t$ as $\mu$ or $\mathrm{v}$ decrease. Moreover, these three parameters do not play the same role in the sense that the differences in $\Delta t^{*}$ are much larger when $\mathrm{v}$ is changed rather than $\mu$ or $\ell$.

Figure 7 displays the evolution of $\Delta t^{*}$ as a function of the capillary number $\mathrm{Ca} \sim \mathrm{v} \mu$, for various experimental conditions, when the velocity signal $V_{\ell}(t)$ is computed at $\ell=\ell_{c}$. The first important result is that the capillary number $\mathrm{Ca}$ does not control the temporal correlations of the global velocity $V_{\ell}(t)$. Indeed, $\Delta t^{*}$ evolves very differently with the imposed flow rate $\mathrm{v}$ and viscosity $\mu$. The dashed line goes through experiments performed at different $\mathrm{v}$ but the same $\mu$ and is proportional to a power law $v^{-1.4}$. Experiments at the same $\mathrm{v}$ but different $\mu$ correspond to the dotted line that goes as $\mu^{-0.4}$. Interestingly, both the values as well as the scaling behavior of the temporal correlations $\Delta t^{*}$ of the global velocity signal $V_{\ell}(t)$ measured at $\ell=\ell_{c}$ are similar to the 


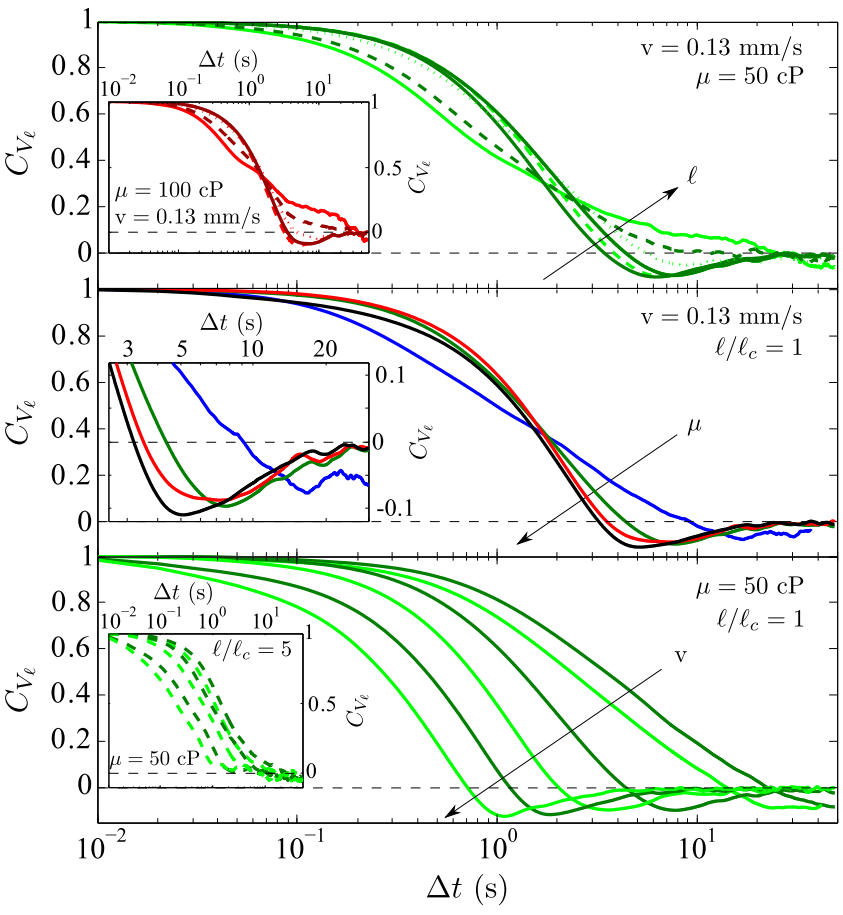

FIG. 6. Autocorrelation functions of $V_{\ell}(t)$ as a function of the time lag $\Delta t$. Top: Evolution with $\ell$ for $\ell / \ell_{c}=10,5,2,1,0.75,0.5$ and two different values of $\mu$. Middle: Evolution with $\mu=10,50$, $100,169 \mathrm{cP}$ for $\ell / \ell_{c}=1$. A zoom of the minima is also shown. Bottom: Evolution with $\mathrm{v}$ from 0.036 to $0.55 \mathrm{~mm} / \mathrm{s}$ for two values of $\ell / \ell_{c}$.

ones of the local velocity reported in Part I (accompanying paper [20]). Those temporal correlations correspond actually to the maximum duration of local avalanches, which follow consistently the scaling $\Delta t^{*} \sim \ell_{c}^{H} / \mathrm{v}$, where $H=0.82(2)$ is the scaling exponent characterizing the shape of the local avalanches. Nevertheless, for $\ell>\ell_{c}, \Delta t^{*}$ becomes larger, as shown in the inset of the figure. At these scales (larger than $\ell_{c}$ ) simultaneous overlapping local avalanches-correlated or not-contribute to the global advancement of the front. The

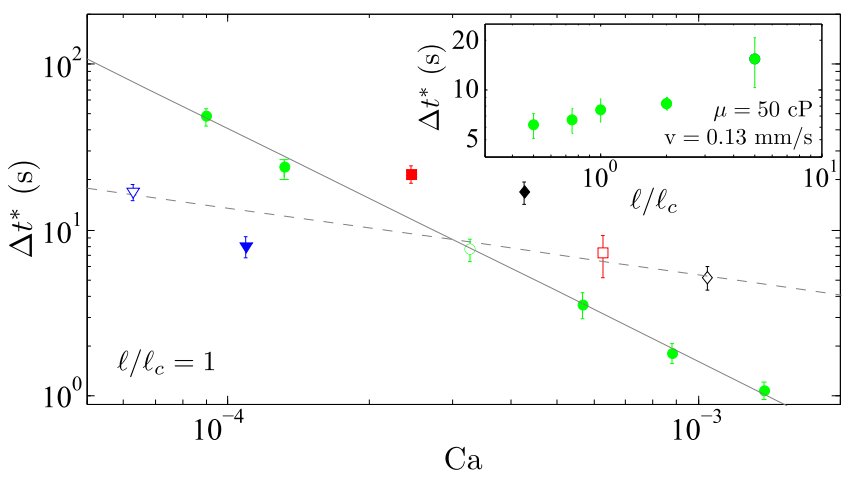

FIG. 7. Evolution of the maximum anticorrelation time $\Delta t^{*}$ with the capillary number $\mathrm{Ca}$. The solid line $\Delta t^{*} \sim \mathrm{v}^{-1.4}$ follows the results for various velocities but one viscosity $\mu=50 \mathrm{cP}$ (solid circles). The dashed line $\Delta t^{*} \sim \mu^{-0.4}$ passes through data for various viscosities but one velocity $\mathrm{v}=0.13 \mathrm{~mm} / \mathrm{s}$ (open symbols). Inset: Evolution with $\ell / \ell_{c}$ for one set $(\mu, \mathrm{v})$. proportionality between $\Delta t^{*}$ and the maximum duration of a local avalanche $\ell_{c}^{H} / \mathrm{v}$ may not hold then. The inset shows also that, for $\ell<\ell_{c}, \Delta t^{*}$ becomes slightly smaller. This is the result of observing local bursts through windows $\ell<\ell_{c}$ which may cut them laterally.

\section{GLOBAL AVALANCHES}

Events of fast motion of the average front in a window $\ell$ can be distinguished from slower advancements. Global avalanches - excursions of $V_{\ell}(t)$ above an arbitrary threshold $V_{c}$-have a size $S$ and duration $T$ defined by the area enclosed and the time elapsed between two consecutive threshold crossings of the signal $V_{\ell}(t)$, as shown in Fig. 8. The size $S$ represents the extra advancement of the front with respect to the distance advanced at velocity $V_{c}$. The threshold used is $V_{c}=\left\langle V_{\ell}(t)\right\rangle+c \sigma_{V}$, where $\left\langle V_{\ell}(t)\right\rangle$ is the temporal average of the signal over the duration of the experiment, $\sigma_{V}$ its standard deviation, and $c$ is an arbitrary constant.

To avoid artifacts arising from the temporal resolution of image acquisition $\delta t$, we will only consider avalanches longer than $T_{\min }=10 \delta t$. The size of the disorder patches also introduces a cutoff size for the smallest avalanches, $S_{\min }$, which we assign to the avalanche produced by the advancement of the front over a single patch of disorder. We have $S_{\min }=\left(\ell_{p} / \ell\right) r$, where $\ell_{p}=0.4 \mathrm{~mm}$ is the lateral size of a disorder patch, $\ell$ is the lateral size of the window of observation, and $r=0.106$ $\mathrm{mm}$ (per pixel) is the spatial resolution.

We first check the effect of the arbitrary threshold $V_{c}$ on the statistical properties of global avalanches. Figure 9 shows the distributions of sizes and durations for different values of $c$. Both $P(S)$ and $P(T)$ display a power-law regime with an upper cutoff. We observe that the clip level $c$ does not affect the power-law scaling of the distributions but shifts the cutoff. Distributions corresponding to the smallest value displayed ( $c=-0.5$ ), however, start to show deviations in the power-law regime that would lead to a lower value of the exponent. For this reason we will consider clip levels larger than $c=-0.5$. Even though all the analysis presented below corresponds to a single value $c=0$, we have checked the robustness of the

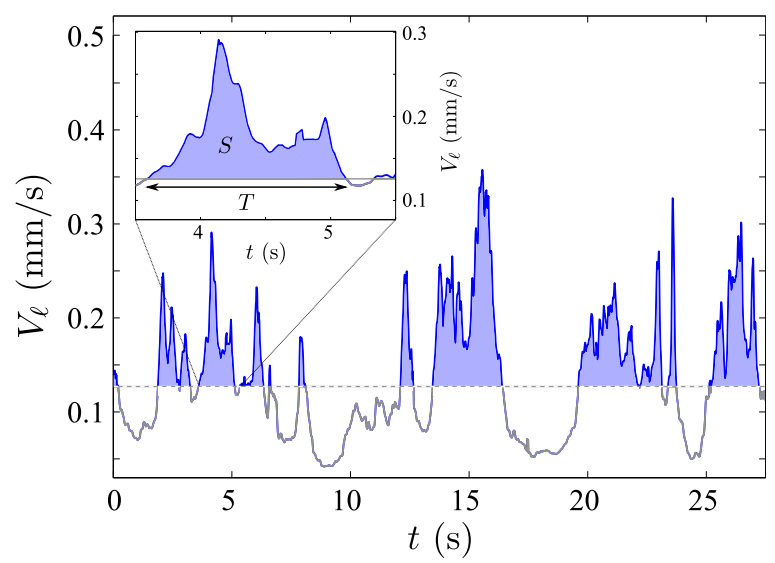

FIG. 8. The main panel shows an example of the global velocity signal for $\ell=L$, clipped by its average value. The inset is a single avalanche of size $S$ and duration $T$. 


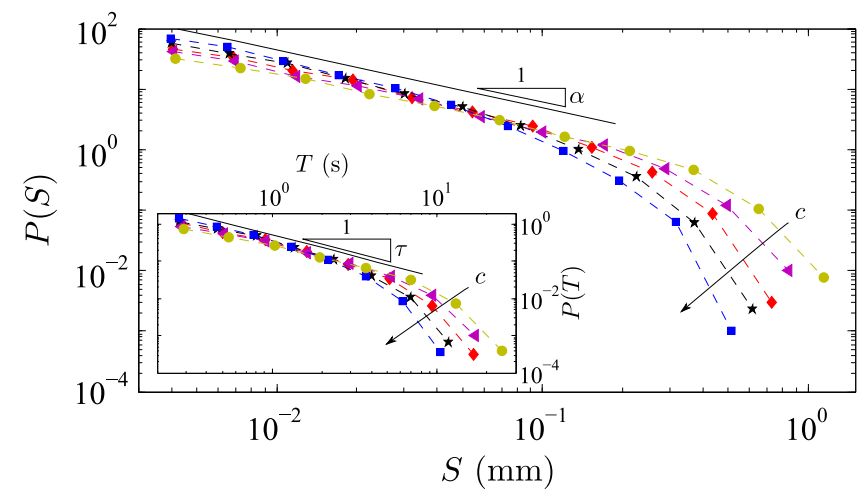

FIG. 9. Effect of the clip level $c$ on $P(S)$ (main plot) and $P(T)$ (inset) for experiments with $\mathrm{v}=0.13 \mathrm{~mm} / \mathrm{s}, \mu=50 \mathrm{cP}$, and $\ell=2 \ell_{c}$. Here $c=-0.5,-0.25,0,0.25,0.5$. Solid lines are guides to the eye corresponding to a power law with exponent $\alpha=0.96$ for sizes and $\tau=1.15$ for durations.

results presented in this section for various clip levels from -0.25 to 0.5 .

\section{A. Influence of the experimental conditions on the statistics of avalanches}

In this section the statistics of avalanche sizes and durations are studied as a function of the experimental controlling parameters, $\mathrm{v}$ and $\mu$, and the observation scale $\ell$.

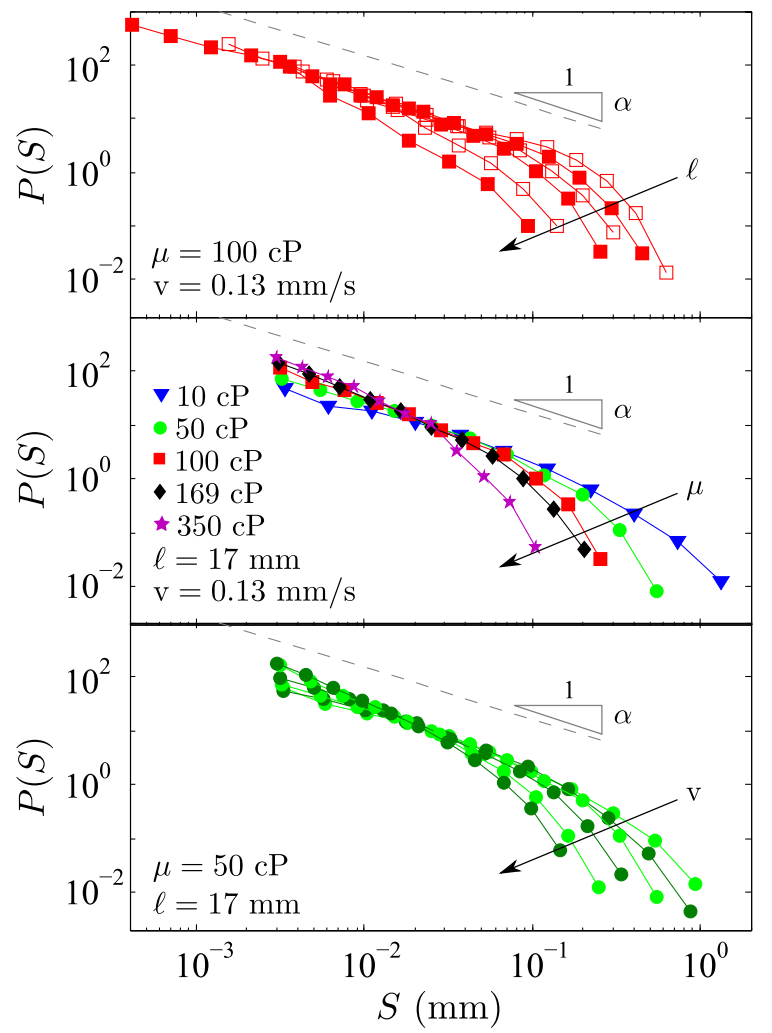

\section{Spatially averaged velocities measured at scales larger than $\ell_{c}$}

Figure 10 shows the evolution of the distributions of sizes (left) and durations (right) with $\ell, \mu$, and $\mathrm{v}$ (top, middle, bottom) when the other two parameters are fixed. In all cases $P(S)$ and $P(T)$ can be represented by the general functional form $P_{x}(x)=a_{x} x^{-m_{x}} e^{-x / \xi_{x}}$ corresponding to a power-law distribution with an exponential cutoff.

The power-law exponent for the sizes of the avalanches is the same irrespective of the experimental conditions or the window of observation. However, the cutoff of the distributions, which controls their scaling range, evolves with the three parameters. The smaller $\ell, \mu$, or $\mathrm{v}$, the larger the power-law range. The dependence of the cutoffs on the controlling parameters can be rationalized in terms of the ratio $\ell / \ell_{c}$, which provides an estimation of the effective number of statistically independent domains of the interface considered to compute $V_{\ell}(t)$. $\ell_{c}$ diverges as $\mathrm{v} \rightarrow 0$ or $\mu \rightarrow 0$. When approaching this limit, the correlation length becomes larger and, therefore, local avalanches occupy a larger fraction of the window of observation. The bigger the underlying local avalanches, the larger the extra advancement of the interface within a given window $\ell$ and hence the size $S$ of the global avalanches. Similarly, when $V_{\ell}(t)$ is computed at progressively smaller scales $\ell$, correlated regions of the front occupy a larger fraction of the window of observation. We expect therefore that the sizes of global avalanches will only depend on the ratio $\ell / \ell_{c}$. This hypothesis will be verified in Sec. III C.

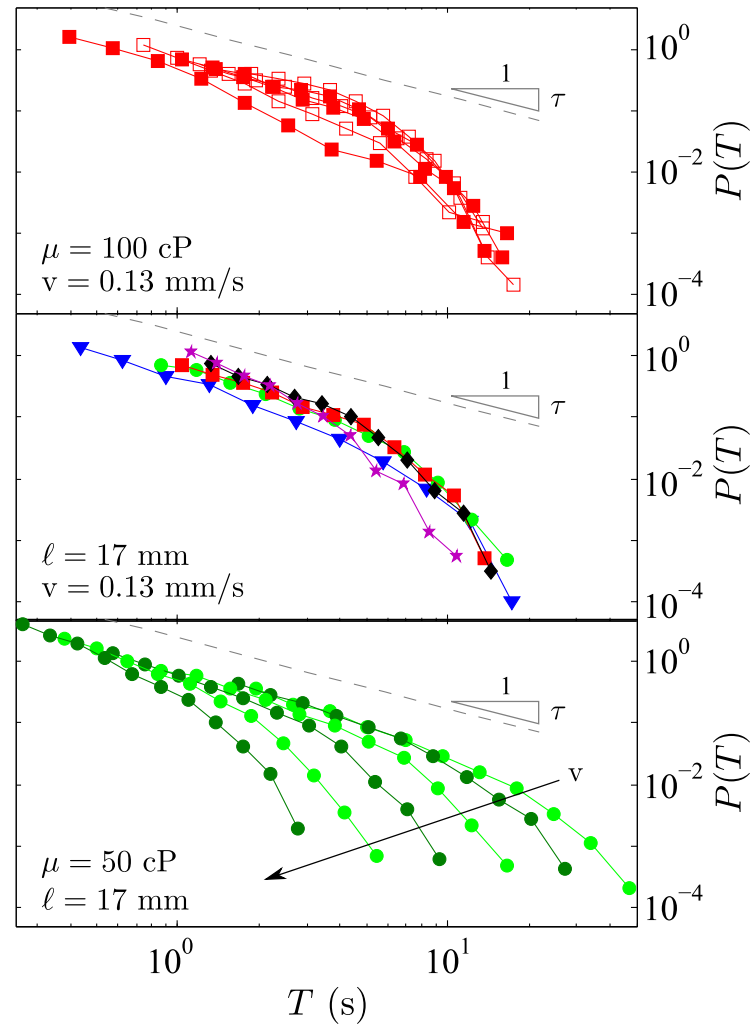

FIG. 10. Distributions of sizes (left) and durations (right) of the global avalanches for velocities analyzed at $\ell \geqslant \ell_{c}$. The top, middle, and bottom panels show the evolution of the distributions with $\ell, \mu$, and $\mathrm{v}$ respectively when the other two parameters are not changed. The top panels show the results for $\ell=136,34,17,12.4,8.5$, and $5 \mathrm{~mm}$ and the bottom panels for $\mathrm{v}=0.036,0.053,0.13,0.23,0.35,0.55 \mathrm{~mm} / \mathrm{s}$. Dashed lines are guides to the eye corresponding to power laws with exponents $\alpha=0.96$ for sizes and $\tau=1.15$ for durations. 

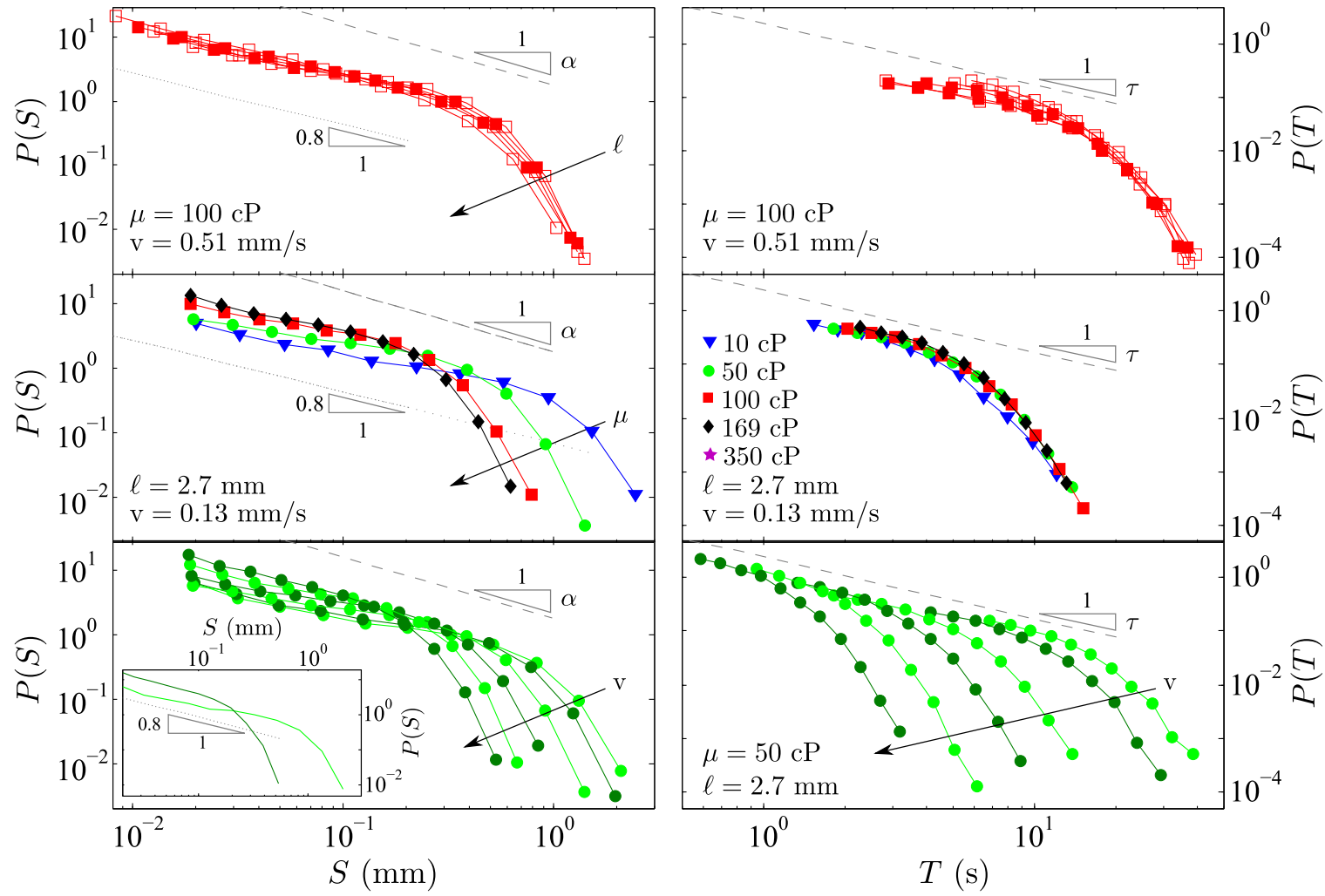

FIG. 11. Distributions of sizes (left) and durations (right) of the global avalanches for velocities computed at $\ell<\ell_{c}$. The top, middle, and bottom panels show the evolution of the distributions with $\ell, \mu$, and $\mathrm{v}$ respectively when the other two parameters are not changed. The top panels show results for $\ell=6.5,5,4.3,3.4$, and $2.7 \mathrm{~mm}$, and the bottom panels for $v=0.036,0.053,0.13,0.23,0.35,0.55 \mathrm{~mm} / \mathrm{s}$. The inset shows the results for the lowest and highest velocity in the main plot. Dashed lines are guides to the eye corresponding to power laws with exponents $\alpha=0.96$ for sizes and $\tau=1.15$ for durations and dotted lines a power law with exponent 0.8 .

Regarding the distributions of durations, $P(T)$, they follow also the same power law at the scaling regime regardless of the controlling parameters. In this case, however, in contrast with the distributions of sizes, the cutoffs of the distributions of durations do not evolve with $\ell$ and $\mu$, but they do evolve with $\mathrm{v}$. The cutoffs of the pdfs, which provide an estimation of the maximum duration of the global avalanches, increase as $\mathrm{v} \rightarrow 0$. This reveals that avalanches behave differently in space than in time. The evolution of the cutoffs is analyzed in more detail also in Sec. III C.

\section{Spatially averaged velocities measured at scales smaller than $\ell_{c}$}

The evolution of the distributions of sizes and durations of global velocities computed at scales smaller than the correlation length are shown in Fig. 11. Distributions of sizes (left panels of the figure) can be approximated again by a power law with an exponential cutoff. However, the value of the exponent $\alpha$ compatible with the data is now smaller $(\simeq 0.8)$. In addition, the distributions show a small bump near the cutoff before the decay. Those two observations are signatures of windowed data, i.e., that local avalanches are cut laterally when the global signal is computed [27].
The evolution of $P(S)$ with $\ell$ is tiny but systematic (top left panel of Fig. 11). The cutoffs are slightly larger at small windows of observation. The effect of $\mu$ and $\mathrm{v}$ is more clear (two other left panels of the figure). The cutoffs increase when either $\mu$ or $\mathrm{v}$ decrease. The behavior of the bump of the distributions is also systematic with the three parameters. Again, the effect is more evident for small $\mu$ and $\mathrm{v}$ because $\ell_{c}$ is larger; the data are more heavily windowed, and therefore the bump is more pronounced.

Distributions of durations of the global avalanches are shown in the right panels of Fig. 11. They can be approximated also by a power-law distribution with an exponential cutoff. In this case the (small) scaling regime is compatible with the value of $\tau$ obtained for $\ell>\ell_{c}$. Moreover, the bump observed in $P(S)$ close to the cutoffs is less clear in these distributions. This reveals that data windowing affects much less the avalanche durations. As it happened for $\ell>\ell_{c}$, the cutoffs of the duration distributions do not evolve with $\mu$ and $\ell$, but they do evolve with $\mathrm{v}$. This shows, once again, that the influence of $\mathrm{v}$ and $\mu$ on the properties of the global avalanches differs in space and in time.

In order to avoid spurious effects arising from data windowing, in the following section, we will consider avalanches of $V_{\ell}(t)$ corresponding only to windows of observation $\ell>\ell_{c}$. 


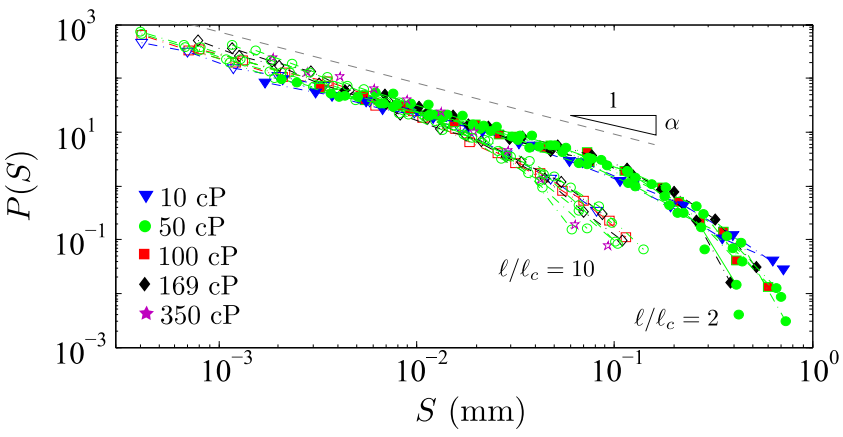

FIG. 12. Distributions of avalanche sizes for $\ell / \ell_{c}>1$. Experiments compatible with $\ell / \ell_{c}=2$ (solid symbols) and $\ell / \ell_{c}=10$ (open symbols) are shown. $\mu$ ranges from 10 to $350 \mathrm{cP}$ and $\mathrm{v}$ from 0.036 to $0.55 \mathrm{~mm} / \mathrm{s}$. The dashed line gives a power law exponent $\alpha=0.96$.

\section{B. Dependence on $\ell / \ell_{c}$ of the distributions of sizes and durations}

Since the fluctuations of $V_{\ell}(t)$ are controlled by the ratio $\ell / \ell_{c}$, as shown in Sec. II, it is natural to study the statistics of avalanches of the signal $V_{\ell}(t)$ from all experiments together at windows of observation such that $\ell / \ell_{c}$ is constant.

Figure 12 shows the distributions of avalanche sizes for data corresponding to $\ell / \ell_{c}=2$ and 10 . As expected, all the distributions show a power-law regime with the same exponent $\alpha$. In addition, experiments performed at different $\mathrm{v}$ or $\mu$ but analyzed at given $\ell / \ell_{c}$ decay exponentially with a very similar cutoff for large $S$. The scaling range of the distributions is controlled by the ratio $\ell / \ell_{c}$ : the smaller $\ell / \ell_{c}$ the larger the cutoff $\xi_{S}$. However, tiny differences for different experimental conditions analyzed at the same $\ell / \ell_{c}$ are still observed.

As for $P(T)$, shown in Fig. 13, we recover a power-law distribution with an exponential cutoff. Again, the power-law exponent does not depend on $\ell / \ell_{c}$. The distributions show no clear dependence on $\ell / \ell_{c}$, either. However, the cutoff $\xi_{T}$ seems to evolve with the imposed mean velocity. The maximum duration of the avalanches gets longer when the fluid is injected more slowly in the system. We will return to the behavior of the cutoffs in Sec. III C.

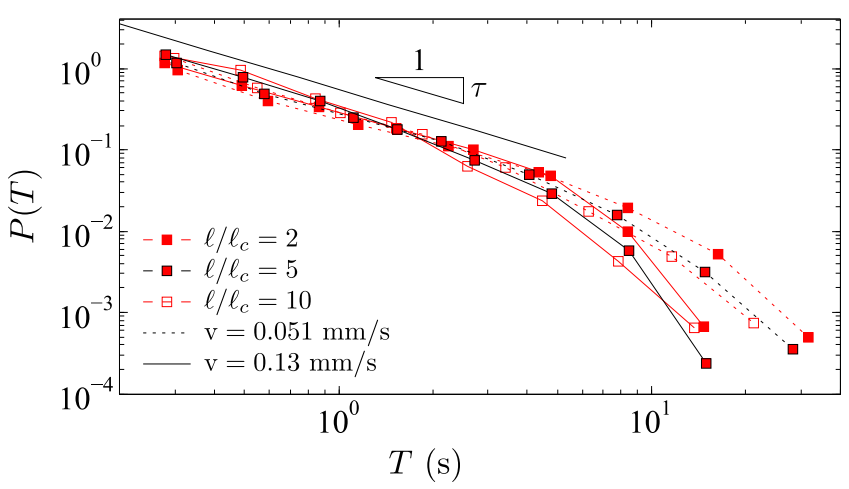

FIG. 13. Distributions of avalanche duration for two experimental conditions: $\mu=100 \mathrm{cP}$ and $\mathrm{v}=0.051,0.13 \mathrm{~mm} / \mathrm{s}$, for three different values of $\ell / \ell_{c}$. The line gives a power-law exponent $\tau=1.15$.

\section{Collapse of the distributions and determination of the power-law exponents}

To obtain accurate values of the power-law exponents, we recompute the statistical distributions of sizes and durations in terms of reduced variables, as explained in Refs. [28,29].

Figure 14 shows the rescaled distributions of sizes (top) and durations (bottom) and their respective fits for experiments analyzed at $\ell>\ell_{c}$. Thirty-four different data sets $(\mu, \mathrm{v}, \ell)$ are considered, showing a remarkable data collapse for both sizes and durations. Experiments at the highest mean velocities, $\mathrm{v}=0.35,0.55 \mathrm{~mm} / \mathrm{s}$ for the oil of $\mu=50 \mathrm{cP}$, and experiments with the oil of largest viscosity, $\mu=350 \mathrm{cP}$, have not been considered in the data collapse. The distributions in these cases are dominated by the exponential cutoff, making the method used to extract $\alpha$ and $\tau$ inaccurate. Nevertheless, the exponents obtained considering these faster experiments or using the largest viscosity are still compatible with the values shown in the figure. The power-law regimes are robust and extend for almost two decades of sizes and durations. However, small deviations at large values of the variables-where statistics is poorer-are observed. The error functions $\epsilon$ and their minima are shown in the insets. From these data collapses we can extract reliable size and duration exponents:

$$
\alpha=0.96 \pm 0.05, \quad \tau=1.15 \pm 0.15 .
$$

These values are in excellent agreement with previous results obtained in the same setup, i.e., $\alpha=1.00 \pm 0.15$ and
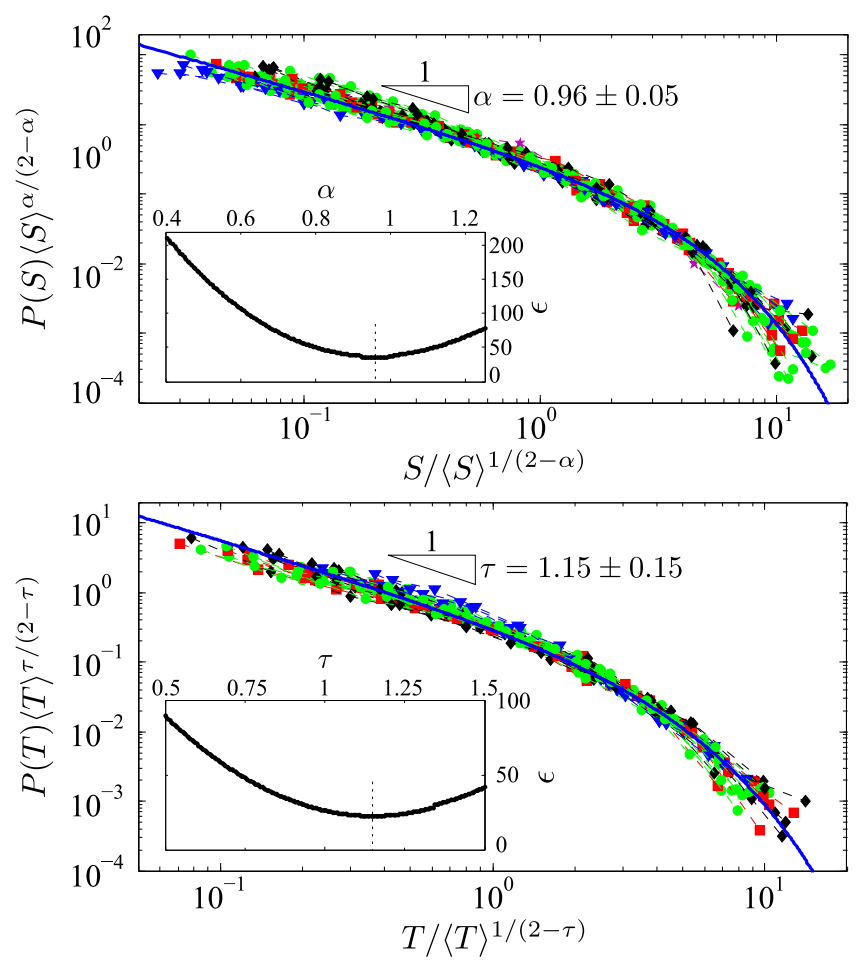

FIG. 14. Data collapse of the probability distributions of sizes (top) and durations (bottom) of the global avalanches for experiments analyzed at $\ell / \ell_{c}>1$. Different symbols correspond to different viscosities. The best fits to a power law with an exponential cutoff are achieved with $\alpha=0.96$ and $\tau=1.15$ (solid lines). The insets show the error of the fits as a function of $\alpha$ or $\tau$. 

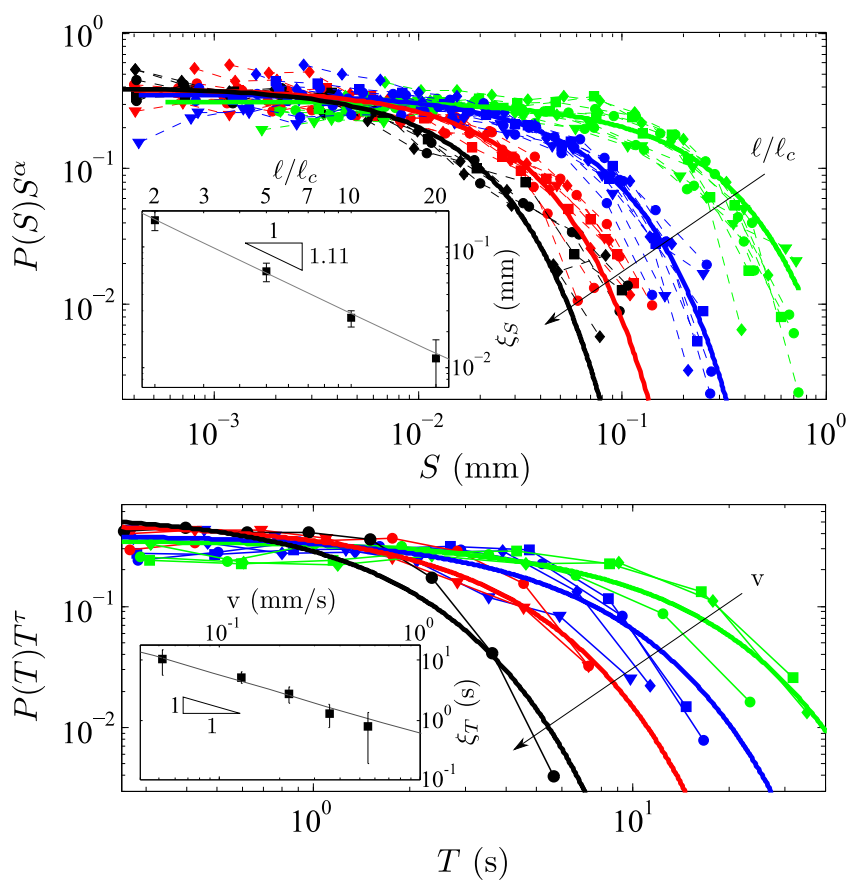

FIG. 15. Top: Distributions of avalanche sizes rescaled by $S^{\alpha}$ for $\ell / \ell_{c}=2,5,10,20$. Solid lines are exponential fits of the data for each $\ell / \ell_{c}$. Bottom: Distributions of avalanche durations rescaled by $T^{\tau}$ for $\ell / \ell_{c}=2$ and $\mathrm{v}=0.052,0.13,0.22,0.35 \mathrm{~mm} / \mathrm{s}$. In both cases different symbols correspond to experiments with different viscosities-we do not distinguish different $\mathrm{v}$ for the same $\mu$. Solid lines are exponential fits of the data for each v. Insets: Evolution of the cutoff values obtained from the previous fits with $\ell / \ell_{c}$ (top) and $\mathrm{v}$ (bottom). Solid lines are guides to the eye.

$\tau=1.25 \pm 0.25[14,30]$. They are now determined with higher precision and, more importantly, they include now experiments with different oil viscosities, showing that the scale-invariant picture of the dynamics that emerges from the previous analysis is very robust. The results also agree with phase-field simulations of constant flow rate imbibitions, where $\alpha=1.03 \pm 0.05$ and $\tau=1.10 \pm 0.11$ were found [31].

Once the the power-law exponents of the distributions have been extracted, we can obtain the cutoffs $\xi_{S}$ and $\xi_{T}$ by considering only their exponential decay. In order to do so, we fit an exponential function to the distribution $P(x) x^{m_{x}}$ versus $x$. The exponential decays of $P(S)$ are displayed in the top panel of Fig. 15. Distributions for different experiments $(\mu, \mathrm{v})$ are grouped by $\ell / \ell_{c}$, as previously observed in Fig. 12 . Small deviations of the cutoffs are still observed. These small fluctuations, however, do not evolve systematically with any of the parameters, indicating that they result probably from lack of statistics. The functions $P(S) S^{\alpha}$ are well fitted by a decaying exponential, from which the cutoffs $\xi_{S}$ are obtained. The inset displays the divergence of $\xi_{S}$ [corresponding to an increase of the power-law range of $P(S)$ ] as $\ell$ approaches $\ell_{c}$ from above, revealing that the system becomes more and more critical. The cutoffs of the distributions of durations are extracted from the curves $P(T) T^{\tau}$ versus $T$. The bottom panel of Fig. 15 displays the results for experiments analyzed at $\ell / \ell_{c}=2$. Distributions are sorted by the imposed mean velocity. The slower the fluid is injected, the larger the cutoff. We find that $\xi_{T} \sim 1 / \mathrm{v}$, as shown in the inset.

The evolution with $\ell / \ell_{c}$ and $\mathrm{v}$ of the mean size $\langle S\rangle$ and mean duration $\langle T\rangle$ of the avalanches parallels the corresponding evolution of the cutoffs. This allows us to characterize the distributions without having to specify their actual shape, which is especially convenient for $\ell<\ell_{c}$, where the distributions display a bump close to the beginning of the decay. The comparison of the cutoffs $\xi_{S}$ and $\xi_{T}$ to $\langle S\rangle$ and $\langle T\rangle$ is shown in Fig. 16. Cutoffs are an upper limit for sizes and durations, and thus their values are larger than $\langle S\rangle$ and $\langle T\rangle$. However, the evolution of both $\langle S\rangle$ and $\xi_{S}$ is perfectly compatible with $\left(\ell / \ell_{c}\right)^{-1.1}$ for $\ell / \ell_{c} \geqslant 1$. As for the durations, $\langle T\rangle$ and $\xi_{T}$ are both consistent with a scaling $1 / \mathrm{v}$. In addition, these durations are compared to the time needed to advance a distance $\ell_{d}$ (the characteristic size of the disorder) at the imposed mean velocity, $\tau_{c}=\ell_{d} / \mathrm{v}$. Although the cutoffs of $P(T)$ depend on the velocity threshold $V_{c}$ chosen for defining avalanches, they are of the same order of magnitude than $\tau_{c}$. Specifically, for the value of $V_{c}$ chosen in Fig. 16 they are equal within error bars. This reveals that the maximum durations of the avalanches of the global velocity $V_{\ell}$ seem to be controlled by the spatial extent of the disorder.

\section{Joint distribution $P(S, T)$}

The distributions of sizes and durations $P(S)$ and $P(T)$ are the marginal pdfs of a joint distribution $P(S, T)$. Figure 17 shows the joint distribution for the rescaled variables $S^{*}=S /\langle S\rangle^{1 /(2-\alpha)}$ and $T^{*}=T /\langle T\rangle^{1 /(2-\tau)}$, which depend on the exponents $\alpha$ and $\tau$ previously obtained, for $\ell / \ell_{c}=1$ and 10 (left and right). In both cases the crest of the joint distribution follows a power law, limited by an upper and lower cutoff indicated by the vertical dashed lines. The upper cutoff reflects the upper cutoffs of $P(S)$ and $P(T)$. The lower cutoff is associated with the minimum possible size of a global avalanche and the discretization of time.

In the scaling regime the most probable values follow $S \sim T^{\gamma}$. We have obtained $\gamma$ from a fit of the whole data
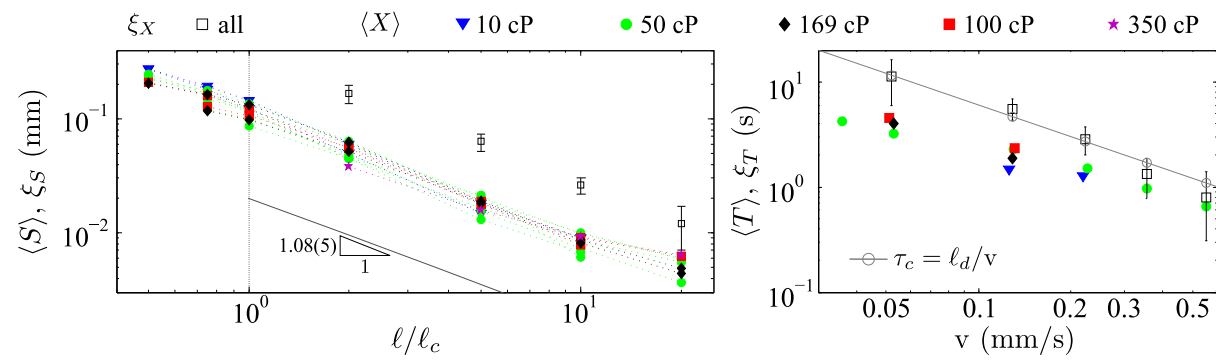

FIG. 16. Dependence of the mean value and the cutoff of the distributions of sizes (left) and durations (right) on $\ell / \ell_{c}$ and $\mathrm{v}$, respectively. The solid line in the left panel is a power-law fit to $\langle S\rangle$ vs $\ell / \ell_{c}$ down to $\ell=\ell_{c}$. In the right panel, the experimental values are compared to $\tau_{c}=\ell_{d} / \mathrm{v}$ with $\ell_{d}=0.6 \mathrm{~mm}$. 


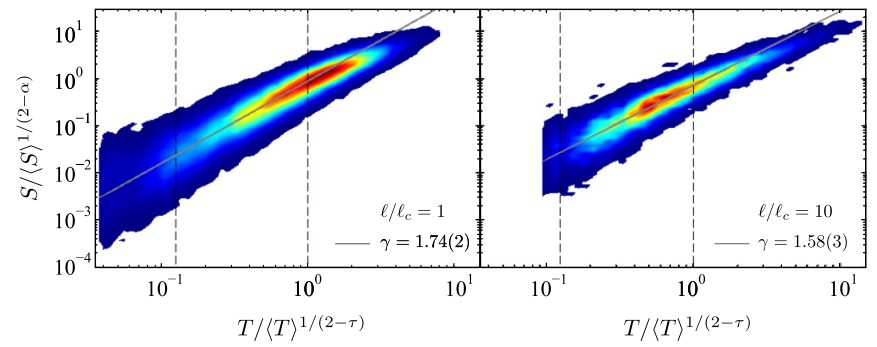

FIG. 17. Probability density plots of the joint distribution of rescaled variables $S^{*}$ and $T^{*}$ for $\ell / \ell_{c}=1$ (left) and 10 (right). Vertical dashed lines show the scaling regime chosen to extract the power-law exponent $\gamma$. The solid straight lines are the best fits $S^{*} \sim\left(T^{*}\right)^{\gamma}$ (statistical error of the fit quoted in the figure). The probability density of points increases from the sides to the center.

set. We consider only avalanches within $T^{*}=T /\langle T\rangle^{1 /(2-\tau)} \in$ $\left[10^{-0.9}, 1\right]$ and $T>0.2 \mathrm{~s}$ to ensure that $T_{\min }>10 \delta t$, where $\delta t$ is the temporal resolution. The values of $\gamma$ obtained are given in the figure. The largest value is obtained when the interface is averaged over a window of size $\ell_{c}$. For larger windows the value of $\gamma$ decreases systematically with $\ell / \ell_{c}$. The maximum variation of measured exponents between $\ell / \ell_{c}=1$ and 20, however, is smaller than $10 \%$.

The exponent $\gamma$ can be related to the exponents $\alpha$ and $\tau$ of the distributions of sizes and durations. Since $P(S)$ and $P(T)$ are marginal distributions of the joint probability distribution $P(S, T)$, given by power laws $P(S) \sim S^{-\alpha}$ and $P(T) \sim T^{-\tau}$ up to the cutoff scales $\xi_{S}$ and $\xi_{T}$, it follows that $\tau-1=\gamma(\alpha-1)$. This scaling relation is compatible with the exponents quoted earlier, within error bars. However, since $\alpha$ and $\tau$ are close to 1 , the value of $\gamma$ derived from this scaling relation is subjected to a very large uncertainty.

\section{DISCUSSION AND CONCLUSIONS}

In this work and its companion paper (Part I [20]) we have studied the spatiotemporal dynamics of stable-imbibition displacements, driven at constant flow rate, in a laboratory model of an open fracture. The study has been supported by large data sets, in which the different roles played by the mean front velocity $\mathrm{v}$, the dynamic viscosity of the oil $\mu$, and the lateral size of the window of observation, $\ell$, have been systematically explored [32]. In particular, carrying experiments with oils of different viscosity but same oil-air surface tension has allowed us to uncover dynamic properties of the front that scale differently with $\mathrm{v}$ and $\mu$, and therefore are not simply controlled by the capillary number $\mathrm{Ca} \sim \mu \mathrm{v}$.

As discussed in Part I [20], fluctuations and lateral correlations of local velocities scale indeed with the capillary number Ca. However, temporal correlations depend more strongly on $\mathrm{v}$ than on $\mu$, scaling as $\ell_{c}^{H} / \mathrm{v}$, where $\ell_{c} \sim 1 / \sqrt{\mathrm{v} \mu}$ and $H=0.82(2)$. This is consistent with the behavior that was predicted theoretically and observed experimentally for the duration of the longest-lasting avalanches at local scale [15].

The fluctuations of the spatially averaged velocity, characterized by the probability density function of the amplitudes of the velocity signal $V_{\ell}(t)$, are controlled only by the ratio $\ell / \ell_{c}$ (interpreted as the number of effective degrees of freedom of the invading front, $N_{\text {eff }}$ ), and hence they scale in the same way with $\mathrm{v}$ and $\mu$ and depend only on the capillary number $\mathrm{Ca}$, as it was the case also for the local velocities. The pdfs of normalized velocities are increasingly non-Gaussian at smaller observation scales, reflecting the fact that the signal $V_{\ell}(t)$ is a spatial average of a spatially correlated quantity - the local velocity $v(x, t)$. However, the theoretical reason why the pdfs are very well represented by generalized Gumbel distributions with the skewness of the experimental data remains yet an open question.

As with local velocities, the temporal correlations of $V_{\ell}(t)$ scale differently with $\mu$ and $\mathrm{v}$. When the invading front is viewed at scale $\ell=\ell_{c}$, in particular, we have found the maximum anti-correlation of $V_{\ell}(t)$ to occur at a time $\Delta t^{*} \sim$ $\ell_{c}^{H} / \mathrm{v}$, which corresponds to the same scaling in $\mu$ and $\mathrm{v}$ that was observed for the temporal correlations of the local velocity in Part I [20]. However, when the scale of observation does not coincide with the lateral correlation length $\ell_{c}$, the situation becomes more complicated. The characteristic anticorrelation time $\Delta t^{*}$ becomes slightly larger when the front dynamics is analyzed at larger scales. We believe that deviations from the expected scaling $\ell_{c}^{H} / \mathrm{v}$ may reflect the presence of lateral correlations between local avalanches at scales larger than $\ell_{c}$. Large-scale correlations have been predicted theoretically indeed for constant flow rate displacements, as a consequence of the global mass conservation per unit time imposed by this particular driving condition [2].

Our experimental setup has the advantage of providing access in detail to the front motion at all scales. This contrasts with other experimental realizations of slowly driven extended systems with avalanche dynamics, where only a spatially averaged temporal signal (a crackling noise) can be recorded.

By changing the scale of observation systematically, we have been able to perform the scale-dependent statistical analysis of the front dynamics reported in this second paper. Our results show that the dynamics of stable imbibition fronts evolve systematically with the observation scale. We already discussed the evolution of the temporal correlations of $V_{\ell}(t)$ with $\ell$. Focusing now on the properties of global avalanches-positive excursions of $V_{\ell}(t)$ above a prescribed threshold-we have found that the distributions of sizes $S$ and durations $T$ follow a power law with an exponential cutof only for $\ell>\ell_{c}$. The power-law exponents $[\alpha=0.96(5)$ for sizes and $\tau=1.15$ (15) for durations] appear independent of the experimental conditions, but they probably depend slightly on the scale of observation. Indeed, the exponent $\gamma$ characterizing the scaling behavior of the joint distributions of sizes and durations $S \sim T^{\gamma}$ slightly evolves with $\ell / \ell_{c}$. In order to verify carefully this result, and try to get a better understanding of this slight evolution, we are currently analyzing the scaling behavior of the average shape of the global avalanches, as recently done in the context of the crackling dynamics of interfacial crack fronts [33]. Such analysis will allow to go beyond the (simple but subjected to large dispersions) measurements of power-law exponents [34]. Additional evidence that the avalanche dynamics of stable imbibition fronts differs at small and large scales is provided by the different values of $\gamma$ and $\gamma_{\mathrm{av}}$, the exponents characterizing the scaling behavior of the joint distributions of sizes and durations of global and local avalanches respectively. 
Interestingly, however, the value of the exponent $\gamma$ measured at the observation scale $\ell=\ell_{c}$ corresponds to the value of the exponent $\gamma_{\mathrm{av}}$. The upper cutoffs of the distributions of sizes and durations of global avalanches also evolve through the scales of observation, but in different way. The maximum size $\xi_{S}$ depends on $\ell / \ell_{c}$, and hence is controlled by the capillary number, while the maximum duration $\xi_{T}$ depends only on $\mathrm{v}$, the mean velocity of the interface. This last observation points to the existence of a new relevant length scale in the direction of front advancement, as discussed below.

To conclude, the ensemble of results presented in the two parts of this work gives a comprehensive picture of the dynamics of stable imbibition fronts in open fractures. Spatial properties are essentially controlled by the effective number of degrees of freedom of the invading front, through the ratio $\ell / \ell_{c}$, which involves the lateral scale of observation and the capillary number of the displacement. Temporal properties, instead, are related to the direction of advancement of the front and do not depend simply on the capillary number.

We just mentioned that the maximum duration of global avalanches depends only on $\mathrm{v}$, pointing to the existence of a characteristic length scale in the direction of front advancement. We believe that this new length scale could be the characteristic linear extent of the disorder patches, $\ell_{d}$. We arrived to the same conclusion in a recent study of the intermittent properties of $V_{\ell}(t)$ [17]. However, since $\ell_{d}$ was not changed in either study, this possibility remains a conjecture that will require further investigation.

Another open question is the reason why the power-law exponents of sizes and durations of global avalanches reported here are in excellent quantitative agreement with the ones obtained from phase-field simulations [31]. This is in contrast with the lack of quantitative agreement between experiments and phase-field simulations for the distributions of local avalanches distributions, as discussed in Part I [20]. It looks as if spatially averaging the front velocities on scales above the correlation length $\ell_{c}$ would be sufficient to eliminate specific features of the local dynamics that might arise from the particular morphology or extension of the quenched disorder. To solve this question it seems necessary to change the properties of the disorder systematically in both experiments and simulations. We have so far investigated only one single configuration of the disorder.

The avalanche dynamics of imbibition fronts at increasingly large observation scales are ultimately a consequence of the burstlike dynamics at local scale. Interestingly, Laurson, Santucci, and Zapperi [35] could obtain a theoretical relation linking the scaling behavior of the clusters of local high velocity and the global avalanche dynamics of an interfacial crack front. However, such a relation cannot be used for the imbibition process studied here. Indeed, the constant flow rate boundary condition in our experiments imposes a global mass conservation per unit time of the invading fluid. This global conservation-obviously not present in the crack problem-may introduce correlations at large scales (larger than the characteristic length scale $\ell_{c}$ ). Those correlations depend moreover on the distance of the front to the inlet (where the boundary condition applies) [2]. A clear measurement and characterization of such large-scale correlations will be an important step in order to establish a theoretical relation between the scaling behavior of the avalanche dynamics at different spatial scales. This constitutes also a subject of current investigation.

\section{ACKNOWLEDGMENTS}

We thank Ramon Planet for interesting discussions. X.C. acknowledges the financial support of ENS de Lyon through the "Enveloppe attractivité" program and the region RhôneAlpes and of MINECO (Spain) through a FPU AP2009-0839 fellowship. This research received the financial support of MINECO, Spain, Project No. FIS2013-41144-P, and AGAUR (Generalitat de Catalunya), Project No. 2014-SGR-878.
[1] M. Sahimi, Flow and Transport in Porous Media and Fractured Rock, 2nd ed. (VCH, New York, 2011).

[2] M. Alava, M. Dubé, and M. Rost, Imbibition in disordered media, Adv. Phys. 53, 83 (2004).

[3] H. Auradou, J. P. Hulin, and S. Roux, Experimental study of miscible displacement fronts in rough self-affine fractures, Phys. Rev. E 63, 066306 (2001).

[4] H. Schütt and H. Spetzler, Capillary crack imbibition: A theoretical and experimental study using a Hele-Shaw cell, Pure Appl. Geophys. 158, 627 (2001).

[5] E. Pauné and J. Casademunt, Kinetic Roughening in Two-Phase Fluid Flow Through a Random Hele-Shaw Cell, Phys. Rev. Lett. 90, 144504 (2003).

[6] B. Berkowitz, A. Cortis, M. Dentz, and H. Scher, Modeling non-Fickian transport in geological formations as a continuous time random walk, Rev. Geophys. 44, RG2003 (2006).

[7] S. Atis, A. K. Dubey, D. Salin, L. Talon, P. Le Doussal, and K. J. Wiese, Experimental Evidence for Three Universality Classes for Reaction Fronts in Disordered Flows, Phys. Rev. Lett. 114, 234502 (2015).

[8] J. F. Duprat, M. Fermigier, F. Goulaouic, and P. Jenffer, Two-phase flow in an imperfect Hele-Shaw cell -influence of wettability defects on interface dynamics C. R. Acad. Sci. 314, 879 (1992).

[9] T. Delker, D. B. Pengra, and P.-z. Wong, Interface Pinning and the Dynamics of Capillary Rise in Porous Media, Phys. Rev. Lett. 76, 2902 (1996).

[10] A. Dougherty and N. Carle, Distribution of avalanches in interfacial motion in a porous medium, Phys. Rev. E 58, 2889 (1998).

[11] M. Rost, L. Laurson, M. Dubé, and M. Alava, Fluctuations in Fluid Invasion into Disordered Media, Phys. Rev. Lett. 98, 054502 (2007).

[12] M. Pradas, J. M. López, and A. Hernández-Machado, Avalanche dynamics in fluid imbibition near the depinning transition, Phys. Rev. E 80, 050101 (2009). 
[13] J. M. López, M. Pradas, and A. Hernández-Machado, Activity statistics, avalanche kinetics, and velocity correlations in surface growth, Phys. Rev. E 82, 031127 (2010).

[14] R. Planet, S. Santucci, and J. Ortín, Avalanches and NonGaussian Fluctuations of the Global Velocity of Imbibition Fronts, Phys. Rev. Lett. 102, 094502 (2009).

[15] S. Santucci, R. Planet, K. J. Måløy, and J. Ortín, Avalanches of imbibition fronts: Towards critical pinning, Europhys. Lett. 94, 46005 (2011).

[16] P. de Anna, T. Le Borgne, M. Dentz, A. M. Tartakovsky, D. Bolster, and P. Davy, Flow Intermittency, Dispersion, and Correlated Continuous Time Random Walks in Porous Media, Phys. Rev. Lett. 110, 184502 (2013).

[17] X. Clotet, J. Ortín, and S. Santucci, Disorder-Induced Capillary Bursts Control Intermittency in Slow Imbibition, Phys. Rev. Lett. 113, 074501 (2014).

[18] S. Whitaker, The Method of Volume Averaging: Theory and Applications of Transport in Porous Media (Kluwer, The Netherlands, 1999).

[19] J. P. Sethna, K. A. Dahmen, and C. R. Myers, Crackling noise, Nature 410, 242 (2001).

[20] X. Clotet, J. Ortín, and S. Santucci, Experimental study of stable imbibition displacements in a model open fracture. I. Local avalanche dynamics, Phys. Rev. E 93, 012149 (2016).

[21] S. T. Bramwell, P. Holdsworth, and J.-F. Pinton, Universality of rare fluctuations in turbulence and critical phenomena, Nature 396, 552 (1998).

[22] E. Bertin, Global Fluctuations and Gumbel Statistics, Phys. Rev. Lett. 95, 170601 (2005).

[23] S. T. Bramwell, The distribution of spatially averaged critical properties, Nat. Phys. 5, 444 (2009).

[24] M. Clusel and E. Bertin, Global fluctuations in physical systems: A subtle interplay between sum and extreme value statistics, Int. J. Mod. Phys. B 22, 3311 (2009).
[25] S. Joubaud, A. Petrosyan, S. Ciliberto, and N. B. Garnier, Experimental Evidence of Non-Gaussian Fluctuations Near a Critical Point, Phys. Rev. Lett. 100, 180601 (2008).

[26] B. Portelli, P. Holdsworth, M. Sellitto, and S. T. Bramwell, Universal magnetic fluctuations with a field-induced length scale, Phys. Rev. E 64, 036111 (2001).

[27] Y.-J. Chen, S. Papanikolaou, J. P. Sethna, S. Zapperi, and G. Durin, Avalanche spatial structure and multivariable scaling functions: sizes, heights, widths, and views through windows, Phys. Rev. E 84, 061103 (2011).

[28] G. Pruessner, Comment on Avalanches and Non-Gaussian Fluctuations of the Global Velocity of Imbibition Fronts, Phys. Rev. Lett. 105, 029401 (2010).

[29] R. Planet, S. Santucci, and J. Ortín, Planet, Santucci, and Ortín reply, Phys. Rev. Lett. 105, 029402 (2010).

[30] R. Planet, S. Santucci, and J. Ortín, Roughness and intermittent dynamics of imbibition fronts due to capillary and permeability disorder, J. Contam. Hydrol. 120-121, 157 (2011).

[31] M. Pradas, Avalanches in disordered media: Scaling growth, avalanche dynamics, and microfluidic fronts, Ph.D. thesis, Universitat de Barcelona, 2009.

[32] X. Clotet, Ph.D. thesis, Universitat de Barcelona and ENS de Lyon, 2014, http://hdl.handle.net/10803/284588.

[33] L. Laurson, X. Illa, S. Santucci, K. T. Tallakstad, K. J. Måløy, and M. J. Alava, Evolution of the average avalanche shape with the universality class, Nat. Commun. 4, 2927 (2013).

[34] S. Papanikolaou, F. Bohn, R. L. Sommer, G. Durin, S. Zapperi and J. P. Sethna, Universality beyond power laws and the average avalanche shape, Nat. Phys. 7, 316 (2011).

[35] L. Laurson, S. Santucci, and S. Zapperi, Avalanches and clusters in planar crack front propagation, Phys. Rev. E 81, 046116 (2010). 